\title{
Blockchain technology: The emerging Human Resources challenge
}

Dr. Maria P Michailidis, Professor of Human Resource Management \&

Organizational Behaviour

School of Business

University of Nicosia

Cyprus

\begin{abstract}
The impact that new technologies have on all aspects of our lives, work, and businesses is significant and growing over time. Blockchain and artificial intelligence, among other innovative technologies, are having a profound effect on practically all business functions, including most human resources (HR) tasks. The HR sector is currently facing a variety of challenges as HR departments invest many hours in vetting candidates' applications and authenticating records to decrease the chances of imperfect recruiting. Nowadays, more technologically based processes are utilised for talent search, selection and management, as well as for accumulating, retaining, and integrating new talents into a company. These HR practices are far more effective compared to traditional methods but can also be more expensive if the transactional costs are considered. This paper consists of two parts. Following an introduction, the first part discusses the trust and confidentiality that can be attained, while the second part examines security, fraud prevention and the productivity gained from using blockchain technology in HR activities. Finally, there is a concluding section, discussing the implications of blockchain technology for firms who choose to employ it.
\end{abstract}

Keywords: blockchain technology, blockchain trust, security, fraud, Human Resource (HR) management. 


\section{Introduction}

Innovative business leaders acknowledge that human resource management is a domain that, if properly addressed, can be a strategic asset for organisations. Spence (2018) mentioned that seeking and recruiting the finest personnel is more critical than ever and investing in employee development is crucial for success in business. HR is becoming more and more complex since modern organisations are shifting increasingly towards contingent labour and work practices beyond traditional organisational processes. For example, temporary talent may come together as a team for a specific project, but then these groups will disperse in the future to work on other tasks under different circumstances, conditions and employment. Although this process of assembling and dispersing talent is considered more effective, undoubtedly it is time consuming and risky as it involves finding the right talent, having them accept certain terms/conditions, forming temporary contracts and legally checking for possible misinformation.

Currently, in the era of the internet, the human resources sector is having to deal with a wider range of challenges than ever before. For example, HR departments invest a lot of time linking, screening and verifying candidates' applications, performing validation tests and authenticating records to minimise the risk of deficient recruiting. The main task of recruiters is to link applicants' profiles to various sources such as, direct applications, recruiting agencies and social networking sites. Consequently, resume checks are time consuming (Yi et al., 2020). As Aishwarya (2018) mentioned, the effects of the blockchain system on HR practices are farreaching and extensive. Organisations can set up their blockchain network solutions by using smart contracts to overcome some of the most significant challenges of HR such as validating employees' profiles, reviewing their performance, checking their credentials, but also handling data across numerous job positions, employers and companies. Blockchain's irreversible and protected function encourages employers to begin assessing a prospective applicant by checking their qualifications and prior accomplishments in the relevant sector. For the applicants, the blockchain ledger will assure their employers of their true skills and potential and allow them to accurately reflect their unique abilities and achievements above that of just a letter of recommendation.

In today's world of internet and cloud computing, it is crucial to provide reliable transaction security and a means of checking the source of a value transaction to avoid fraud, hacking and 
ransomware. For this to be successful, stable networks that can be trusted must be established. The accuracy and credibility of the information processed on a daily basis can be tested using the Blockchain technology, functioning as a credible system where information is verifiable, irreversible and immutable. It is an ever-growing archive that safely holds a digital record of all the transactions that have been processed. Yet another advantage Blockchain offers is that each transaction is stored in chronologically (Aishwarya, 2018). The blockchain network revolves around the following unique characteristics as portrayed by Salleh et al. (2019). The first is decentralisation where the system manages to decentralise trust and allocates a healthy flow of information that limits any type of intermediate interaction. Once most of the network members give their authorisation, after receiving sufficient proof that the cryptographically transmitted information is valid, only then can new data be added to the blockchain ledger. The system is also characterised by consistency. Since all transactions are transmitted over the network, authentication and documentation are required for each block of information, therefore an interruption during the transaction is highly unlikely to occur. In addition, each block is validated, and the transaction is tested and confirmed by other nodes. The blockchain ledger is not managed by a single entity (an individual, business or government) but by all network computers participating globally. This implies that there is no need for any central middleman to certify transactions or check that the documents are genuine, therefore, two or more parties can communicate simultaneously. Additionally, the system can generate substantial improvements in cost saving as there is no need for a third party to be involved during agreements or transaction procedures, thus their services are not needed (Makridakis and Christodoulou, 2019).

As described by Yaga et al. (2018), blockchain systems are tamper-evident and tamper-proof virtual records deployed in a distributed manner (no centralised base) and typically without the need for a central authority to validate them. In its primitive form, the blockchain system permits a society of users to document transactions in a shared ledger within that society, where no transaction can be reversed in the blockchain network once it has been approved and recorded. Distributed blockchains are digital ledgers of cryptographically signed transactions that are clustered into blocks. Post verification and firm conclusions, each block is cryptographically connected to the previous one thus making it tamper-proof. When new blocks of information are introduced in the system it is extremely difficult to modify the older blocks of information thus establishing tamper resistance. The idea is that when new blocks of information are added they 
are automatically replicated across the network through copies of the ledger. If any disputes occur from that process, they are automatically resolved by the system through the defined rules that were set in place when the system was formed.

In previous research by Kitsantas, Vazakidis and Chytis (2019), different types of blockchain systems had been discussed. The most important are Public, Private and Consortium Blockchains, each with their own advantages and disadvantages. In detail, in a Public Blockchain anybody can manage transparent transactions on the network anonymously. This type is mostly used for Bitcoin, is completely decentralised and revolves around users' consensus. However, it is prone to cyber-attacks as a hacker can potentially recreate and fully chain all the blocks of information that have been updated without being identified by the users. In a Private Blockchain system, transactions are anonymous and the data is not open to the public, although the users are known. Specifically, without an invitation to enter the network, a user does not have the ability to write or read any information in the Blockchain network. Such a type is typically used by large corporations with defined permissions among stakeholders of the blockchain enterprise. Lastly, a Consortium Blockchain is a hybrid model that consists of both the public and private model. When choosing this model, organisations may have their own personal blockchain network to share the information among the pool of Consortium users, which might include banks, different companies, or corporations.

\section{Trust relations and confidentiality}

Even though Blockchain has infiltrated numerous aspects of our work by redefining the way we interact, manage, execute and authenticate, it initially mostly influenced the areas associated with capital markets and transactions. Gradually though, its impact on the workplace and specifically on HR processes, which is both prevalent and profound, has become extremely valuable.

As was mentioned earlier, it is feasible through this technology to build an economic system that can run autonomously and trust-free. This new encounter with the digitisation of reliance and confidence, is the innovative prelude of a progressive impact on the way workplaces manage people. For example, in the search and selection process of finding qualified candidates for job 
openings, blockchain technology enables peer-to-peer contact, thus not only streamlining the flow of information in the hiring process, but also reducing cost since there are no intermediaries. Consequently, blockchain technology enables job applicants to connect directly with recruiters and at the same time maintain confidentiality. Moreover, a candidate's background can be verified in an immutable way with true information, via an exchange process with a blockchain network. Thus, a significant aspect and one of the unique features of blockchain during transactions is the enforced trust, as the involvement of any third party is eliminated. Ultimately, such technology is acknowledged with reducing operating costs and improving transaction efficiency by reducing the involvement of intermediaries, accompanied by the fundamental benefit of removing any obstacles to building relations.

Therefore, transparency, but also privacy in service, establish a relationship between users and the technology in question that in return allows trust-free business services. The ordinary trust that was placed upon an individual or an organisation is replaced with the trust across an entire population as the network allows communication with several entities (Fremont and Jonathan, 2018), which is expected in HR practices related to hiring. Consequently, this innovative technology will be influential not only in creating a more transparent organisational culture, but also in fostering trust in work relations between employers and employees.

It is inevitable then, that these fast-moving changes in technology have initiated and will continue to bring multiple advanced changes to the HR space. Since HR manage substantial amounts of sensitive information related to the staff and the company itself, a high level of security and confidentiality are at the core of these HR processes making blockchain technology a suitable fit for the HR industry.

In terms of data confidentiality, Nascimento et al. (2019) deliberated that one of the key benefits of the blockchain network is to oversee and validate the data stored in a confidential manner. Any transaction or contract made through the system is time stamped and ensures that records including payment information, contracts, transfer of ownership and others, are publicly connected with the specific date and time they were generated. This is immensely useful and convenient for the different parties involved as it allows them to check when and who initiated a particular transaction or confirm that the data existed at a specific time and date. As a means to 
ensure data authenticity and transparency, Blockchain relies on public-private key cryptography. It is based on the combination of public keys which permit participants on the network to share them and private keys that allow participants to maintain a unique identity. If a recipient does not have the corresponding public key which is shared by the sender to access the private encrypted message or transaction, then no access is allowed. Whereas if the information is encoded with a private key, they can only be deciphered by the recipient's private key.

Furthermore, as far as contracts are concerned, Neiheiser et al. (2019) explain that smart contracts are software programmes which can implement the conditions of deals between parties without any need for intermediaries. Such contracts were constructed to access executable code on the blockchain for potential invocations. This makes it possible to build escrow structures that do not require the participation of intermediaries. The reason for this circumstance is because a contract is automatically enforced on all blockchain nodes when the right conditions have been fulfilled. HR departments claim that transparency is a problem in the hiring process with either temporary or permanent applicants. This process could be hard for job candidates to follow since they cannot be sure of their application status and whether just and impartial non-discriminatory practices are being used in their assessment. Nonetheless, with the use of Blockchain and smart contracts, employment agreements could be a transparent and a decentralised method of processing applications.

Today's HR trend is related to temporary employment agreements; these have a vital role in the world's current economic and social climate. The growing international rivalry, slow economic growth rates and increasing unemployment have raised the demand for labour flexibility in organisations, and the proliferation of nonstandard contractual arrangements, also fuelled by technological innovation. On a global scale, the competition between companies could reflect upcoming challenges and promptly adapt to changes. Therefore, temporary employment can play a key role as a flexible tool for companies to use to compete in the market. Nevertheless, temporary employment raises concerns which include no guarantees towards employees, lower wages, lack of vocational training due to insufficient time, therefore inadequate growth prospects and limited access to social security systems (Pinna and Ibba, 2017). A solution to the increased concerns regarding temporary employment is the use of a blockchain network as it ensures reliability, trust and transparency. Since the data are stored inside a ledger that does not require 
any paperwork, any information inserted into the system is organised chronologically and communicated across the network.

Furthermore, in compliance with the legislation, Blockchain permits the creation of contracts that protect both the employer and employee. Hence these smart contracts are available to be examined for compliance by the authority whenever it is required. The researchers Pinna and Ibba (2017) introduced the Decentralised Employment System (D-ES), a blockchain based system aimed at assisting employers to create fast and temporary contracts that legally protect but also respect the rights of all parties involved. In doing so, based on the smart contracts, the Decentralised Employment System provides the benefit of any agreement and data gathered between the employer and employee being analysed automatically throughout this system and generating the contract. It therefore manages, controls and monitors the accurate execution of an employment contract. This makes drawing up contracts less time consuming, increases accuracy and enforces compliance with legislation, while at the same time protecting the employees.

Ultimately, successfully implementing blockchain can assist in the prevention of both organisational, internal fraud and hacks, and also external hacks of personal employee records related to finance, banking, salaries, healthcare, performance history, cost reimbursement, and disciplinary records to name a few.

\section{Security, fraud prevention and productivity gains}

Authenticating talent acquisition data as explained by Onik et al. (2018), directly impacts the rate, motivation and productivity level of human resource management. During the recruitment process, certain job seekers can mask unpleasant results and present dishonest information to recruiters. Many candidates present inflated resumes in addition to bogus training and graduation certificates, endorsements, awards, promotions and so on, while others intentionally overstate their credentials and skills. Walker's report, "The Hidden Risks of Recruitment" (2017), stated that human resource professionals are often under additional time pressures as their leaders anticipate a broader strategic HR role in the preservation and evolution of the organisation's HR practices, by landing the best new talents. These demanding expectations include recruiting the most suitable personnel and establishing the best possible working conditions for them, however, just a small number of HR departments regard 
themselves as being completely resourced. HR departments have pointed out multiple gaps in organisations' background screening processes. These gaps often place companies at risk of being staffed with inefficient or incompetent employees which results in fewer completed tasks, a lower quality of work, the need of additional support from colleagues, unsatisfied clients, reduced morale and the damaged reputation of the organisation.

When involved in job recruitment, HR teams come across numerous documents which, as noted earlier, may involve the risk of being given false information by candidates. In order to limit the legal and commercial risks resulting from job applicants falsely representing themselves (in terms of former employers, work experience and general credentials), organisations usually employ HR agencies whose job is to verify this information. However, this authentication process, besides being costly and time consuming, may also involve another risk of being paired with a third-party HR agency which is not dependable and honest and does not preserve privacy. Granted, the verification of information during the job recruitment process is crucial, yet it appears that small and medium-sized companies (Sarda et al., 2018) tend to avoid it. However, in their research, Sarda et al. (2018) reveal just how with the use of technology, specifically blockchain, the HR teams can acquire verified information on the job applicants in real-time and in a cost-effective manner. They also support that trustworthiness and protection of privacy (in terms of employment background) are its key features which all HR areas value. Thus, data sharing is secure and verified.

Moreover, referring to the cryptographic manner that communication takes place within the system, blockchain technology offers improved security, specially designed for safeguarding highly sensitive personal information, for instance financial transactions. Blockchain technology's greatest strength is that it not only settles financial transactions, but it can also function as a decentralised, indefinitely unalterable essential platform for just about any piece of data that needs to be added and communicated across a server. Such a distinctive capability makes the technology ideal for all forms of data, transactions, work credentials, and confidential records/documents to be processed and certified (Makridakis and Christodoulou, 2019). For an employee, maintaining their virtual record in HR has several benefits. For example, Sarda et al. (2018) explained that only a current employer can add a new piece of information with the employee's consent to the network. Also, any former or current employer will never be made aware when new information is added by a future employer, nor when their record is accessed. 
After an employee's record has been developed and authenticated in order to be added to the system, the information cannot be modified. Instead, each time a current employer is interested in adding any new information or accomplishments, these will be added chronologically in the form of blocks. Employees can have a continuously updated and verified work record while simultaneously being able to provide potential employers or recruiting corporations with the required selected documents. Lukić et al. (2018) also proposed that the improved blockchain technology makes employment records easily accessible and organised as everything is stored digitally with no paper trail required, thus eliminating the risk of fraud whilst assessing a candidate's record.

Among the beneficial outcomes of an HR blockchain network is the opportunity for productivity gains as it improves the employee's capability to adapt their expertise and results to their work, therefore improving the overall productivity levels of an organisation. According to Sarda et al. (2018), "The Australian Financial Review" estimated that the average time needed to obtain a vacancy in Australia in 2015 was 68 days, which led to a 50\% rise since 2010. Recruiting procedures mainly involve validating a candidate's profile (history background), which can be both time consuming and complex as it involves obtaining information on the candidate from prior employers. The role of a prior employer is to provide a statement of services which mostly includes only the higher level of job attainments. However, the researchers stated several concerns regarding the usual procedure when obtaining information on a candidate. Specifically, they discussed that gathering information is time consuming, as it can take days to track down a reliable source that will provide important information regarding the candidate. Also, prior employers may be unavailable during the time of research or busy with their job responsibilities, and close-down periods interfere with gathering information, particularly in different time zones in case of an international company, therefore, valuable time is lost.

Moreover, recruiters are still at risk of receiving falsified information as the most common means of gathering data about a candidate is through their most recent work environment. To avoid wasting time most recruiters will only contact the most recent employer. Not having a candidate's full background data can be risky at times as they do not have accurate records regarding their entire employment period. For instance, at times of conflict or when any unusual behaviour occurs in the workplace, they have no reference to go on, as the employee's recent 
statement of service most likely only included positive comments. Other difficulties to acquire information arise when the company where a candidate was previously employed has ceased its operation. Hence the blockchain network can provide several benefits for an organisation by helping it to reduce the likelihood of inaccuracies efficiently and effectively. For example, due to the network's complex yet simplistic way of operation, it ensures that once an employee's work history is validated it can be maintained in the system, thus, future employers will not have to search for the information on their own. The system can potentially replace the statement of service with a block of information containing each prior employment a candidate had which can be used as an employment record. The network only offers beneficial outcomes for organisations as it diminishes the standard procedure of gathering information, coupled with being less tedious since it does not require the validation of work history through third parties, but instead it can easily be accessed through the system. Therefore, an individual will have their own virtual organised employment record which they, as well as current and future employers, can safely view at any time. Another benefit offered by the blockchain network is that employees will not lose vital information regarding their work history in case a company ceases its services. As Mercer (2019) supports, Blockchain "also provides employers with the most accurate snapshot of a candidate's credentials and background".

As stated, especially small and medium-sized companies as well as individual owners could benefit the most from this new technology, since they may lack the resources to search for and recruit talent. Moreover, many such businesses tend to underinvest in training programmes and in the general professional development of their employees and of themselves. In addition, when continuously accessing professional programmes, they tend to face challenges such as limited funding and time to exploit available opportunities (Fachrunnisa and Hussain, 2020).

Finally, the current and future skills needed tend to be overlooked due to other, more pressing activities within the organisation. Blockchain can assist with such difficulties as the database can locate needed personnel depending on the job requirements while suggesting ways for employees within organisations to advance their skills and knowledge. Furthermore, the database can provide useful information about the organisation's needs and the specific training that employees or potential candidates need to undergo to fulfil them. This is an automatic activity carried out within the system using available information, thus freeing the HR department from 
having to deal with such responsibilities and resulting in a positive effect in terms of time and productivity. Aishwarya (2018) also mentioned other possible benefits such as, recruiters discovering further details about the applicants than what has been written in their resume, while any comments about praise or criticism found in their ledger cannot be altered or removed. This makes it easier for recruiters to recognise someone in the pool of candidates who is highly qualified and competent. Blockchain also protects employers from 'Resume Polishers' who exaggerate, inflate, or even create false qualifications to maximise their chances of being recruited. Other types of information regarding candidates that are accessible to recruiters via Blockchain are education verification, media and civil records, employer's credit reports, criminal records, sex offender records and driving licenses. Last but certainly not least, blockchain technology can also help standardise career profiles which lead to the improvement of the resume content's quality and record and track an employee's career development over time (Lukić et al., 2018).

\section{Conclusions}

This paper has identified the major challenges and discussed the innovative benefits that new technologies, especially Blockchain, are providing business firms with, which are transforming human resource tasks within organisations. Today's highly competitive, global environment is forcing organisations to adopt cutting-edge technologies. The use of Blockchain, specifically in HR practices and particularly in recruiting, has been documented to be advancing at a fast pace, providing significant benefits to organisations. Whereas Mercer in 2019 estimated that approximately $0.5 \%$ of global firms were using blockchain technology, according to the same source demand is growing rapidly and it is expected that within the next 10 years, $80 \%$ of firms will be utilising some form of blockchain notions. Blockchain's ability to transform business processes is inspiring HR processes and is reportedly helping foster stronger transparency, accuracy and confidence in HR organisational practices. Writer (2018) claims the technical performance of new technologies, specifically blockchain, will not only contribute to operational improvements, but also to "overall systemic risk reduction". Therefore, it is essential that 
companies start exploring Blockchain's features and the possibility of integrating this new technology into their HR processes.

The mainstream adoption of Blockchain by the HR sector could benefit both employers and employees, first by offering hiring managers the opportunity to hire the right people for the right jobs. With time, Blockchains' value will only grow as HR professionals find more ways to exploit it in order to dramatically reduce the difficulties they face in verifying and contracting prospective talent, thus making the task of searching for the right person less troublesome and more efficient. The efficiency and benefits of using blockchain searching in the recruitment process that this paper has advocated is among the main fields of interest that researchers of the new technology are studying and bound to improve.

It is well documented that to avoid fraud, hacking, and ransomware, it is important to use a system that will provide protection while verifying the source of transactions. Moreover, HR departments are currently faced with a wider range of difficulties. In order to reduce the risk of insufficient hiring, departments spend a lot of time identifying, screening, and checking candidates' applications, as well as conducting validation checks and authenticating records. Organisations pay a considerable amount of money to third party companies to recruit suitable personnel, or to intermediaries when forming contracts. Worse, firms spend considerable amounts of money for recruitment and training purposes, only to sometimes find out the hired employee is not a suitable match with the company, obliging them to repeat the search. Hiring the wrong candidate can have serious ramifications for organisations as it reduces morale and productivity while increasing expenses. Avoiding recruitment problems, therefore, can have significant advantages for HR departments that can benefit the entire organisation by assisting recruiters to validate a candidate's profile online in a trustworthy and efficient way, since data is stored in a virtual immutable ledger.

In conclusion, Blockchain can generate substantial gains in terms of improved efficiency, strengthened security, greater traceability and amplified transparency. The numerous potentials for Blockchain in the HR landscape are unfolding at a fast pace and it is up to the professionals involved to consider their significance and exploit their extensive uses to provide competitive advantages for the firms utilising them. As Blockchain becomes more mainstream and more widely accessible, this disruptive technology will greatly influence human resource management practices and become a necessary HR tool used by all successful firms. 


\section{References:}

Aishwarya, N., (2018). Potential impact of Blockchain on HR and People Management. International Journal of Emerging Technologies and Innovative Research, 5(9), pp.126, 128-129 http://www.jetir.org/view?paper=JETIRA006284 (Accessed 19 February 2021).

Al-Khasawneh, A.L. (2021). Employee Selection and Recruitment Contracts in the Digital Transformation Era, Chapter 6, Jordan: Balqa Applied University

do: 10.4018/978-1-7998-4501-0.ch006

Elayan, M.B. (2021). Transformation of Human Resources Management Solutions as a Strategic Tool for GIG Workers Contracting, Saudi Arabia: Institute of Public Administration

Fachrunnisa, O. and Hussain, F., (2020). Blockchain-based human resource management practices for mitigating skills and competencies gap in workforce. International Journal of Engineering Business Management, 12, p.3

https://journals.sagepub.com/doi/10.1177/1847979020966400 (Accessed 19 February 2021)

Fleming, P., (2019). Robots and organization studies: Why robots might not want to steal your job. Organization Studies, 40(1), pp. 23- 38.

Fremont, V. and Jonathan, G. (2018). Can Blockchain Technology Solve Trust Issues in Industrial Networks?. In: 17th International Conference Perspectives in Business Informatics Research. BIR Workshops, p.401

https://www.semanticscholar.org/paper/Can-the-Blockchain-Technology-Solve-Trust-Issues-inFr\%C3\%A9mont-Jonathan/107586b0ab73c88ccaf5f1842c49db437cbed018 (Accessed 19 February 2021).

Kitsantas, T., Vazakidis, A. and Chytis, E., (2019). A Review of Blockchain Technology and Its Applications in the Business Environment. In: International Conference on Enterprise, Systems, Accounting, Logistics \& Management. pp. 4-5

https://www.researchgate.net/publication/334615432_A_Review_of_Blockchain_Technology_a nd_Its_Applications_in_the_Business_Environment (Accessed 19 February 2021).

Lukić, J., Salkić, H. and Ostojić, B., (2018). New job positions and recruitment of employees shaped by blockchain technologies. In: Fourth International Scientific Business Conference LIMEN 2018-Leadership \& Management: Integrated Politics of Research and Innovations. Belgrade: Association of Economists and Managers of the Balkans, p.5. 
https://www.researchgate.net/publication/330959205_NEW_JOB_POSITIONS_AND_RECRUI TMENT_OF_EMPLOYEES_SHAPED_BY_BLOCKCHAIN_TECHNOLOGIES (Accessed 19 February 2021).

Makridakis, S. and Christodoulou, K., (2019). Blockchain: Current Challenges and Future Prospects/Applications. Future Internet, 11(12), p. 3, 6

https://www.mdpi.com/1999-5903/11/12/258 (Accessed 19 February 2021)

McKinsey Global Institute (2017). Jobs lost, jobs gained: Workforce transitions in a time of automation. Brussels: McKinsey.

Mercer (2019). Blockchain in HR: interesting use cases for human resources

https://www.mercer.com/our-thinking/blockchain-for-humanresources.html\#: : :text=While\%20just \%200.5\% 25\%20of\%20the,some\%20capacity\%20within\% 2010\%20years (Accessed 4/12/2021)

Nascimento S. et al. (2019), Blockchain Now And Tomorrow: Assessing Multidimensional Impacts of Distributed Ledger Technologies, Publications Office of the European Union, Luxembourg, pp.17-19

https://publications.jrc.ec.europa.eu/repository/handle/JRC117255 (Accessed 19 February, 2021)

Neiheiser, R., Inácio, G., et al. (2019). HRM Smart Contracts on the Blockchain. In: 2019 IEEE Symposium on Computers and Communications (ISCC). IEEE, p.1

https://ieeexplore.ieee.org/document/8969692 (Accessed 19 February 2021)

Onik, M., Miraz, M. and Kim, C., (2018). A Recruitment and Human Resource Management Technique Using Blockchain Technology for Industry 4.0. Smart Cities Symposium , p.1 https://www.academia.edu/37981374/A_Recruitment_and_Human_Resource_Management_Tec hnique_Using_Blockchain_Technology_for_Industry_4_0 (Accessed 19 February 2021)

Pinna, A. and Ibba, S. (2017). A Blockchain-Based Decentralized System for Proper Handling of Temporary Employment Contracts. Advances in Intelligent Systems and Computing, pp.1-2.

https://www.researchgate.net/publication/328657407_A_Blockchain-

Based_Decentralized_System_for_Proper_Handling_of_Temporary_Employment_Contracts

(Accessed 19 February 2021) 
Salleh, F., Radzi, R., et al. (2019), New Information Management Dimension in Blockchain. International Journal of Academic Research in Business and Social Sciences, 8(12), pp.1384-1385

http://dx.doi.org/10.6007/IJARBSS/v8-i12/5241 (Accessed 19 February 2021)

Samani, H. et al. (2012). Towards robotics leadership: An analysis of leadership characteristics and the roles robots will inherit in future human society, In J.-S. Pan, S.-M. Chen and N. T. Nguyen (eds.), Asian conference on intelligent information and database systems. Berlin: Springer, pp. $158-165$.

Sarda, P., Chowdhury, M., et al. (2018). Blockchain for Fraud Prevention: A Work-History Fraud Prevention System. In: 17th IEEE International Conference on Trust, Security and Privacy in Computing and Communications and 12th IEEE International Conference on Big Data Science and Engineering. Institute of Electrical and Electronics Engineers, pp.1-3

https://www.researchgate.net/publication/327484134_Blockchain_for_Fraud_Prevention_A_Wo rk-History_Fraud_Prevention_System (Accessed 19 February 2021)

Sauppé, A. and Mutlu, B. (2015). The social impact of a robot co-worker in industrial settings. Proceedings of the 33rd Annual ACM Conference on Human Factors in Computing Systems.

https://dl.acm.org/citation.cfm?id=2702181

Scassellati, B. and Hayes, B. (2014). Human-robot collaboration, AI Matters, 1, pp. 22- 23.

Spence, A., (2018). Blockchain And The Chief Human Resources Officer. Transforming the HR Function and the Market for Skills, Talent, and Training. Brightline Initiative and the Blockchain Research Institute.

https://s3.us-east-2.amazonaws.com/brightlinewebsite/downloads/reports/Brightline_Spence_Blockchain-and-the-Chief-Human-ResourcesOfficer_Blockchain-Research-Institute.pdf?utm_source=resource-page\&utm_medium=button (Accessed 19 February 2021).

Walker, D., (2017). The Hidden Risks of Recruitment: How To Recognise Them And Protect Your Organisation. CVCheck, pp.4,9

https://checkpoint.cvcheck.com/white-paper-the-hidden-risks-of-recruitment-how-to-recognisethem-and-protect-your-organisation/ (Accessed 19 February 2021)

Yaga, D., Mell, P., et al. (2018). Blockchain Technology Overview. NIST, pp.1-2, 15 
https://www.nist.gov/publications/blockchain-technology-overview (Accessed 19 February 2021).

Yi, C., Yung, E., Fong, C. and Tripathi, S., (2020), Benefits and Use of Blockchain Technology to Human Resources Management: A Critical Review. International Journal of Human Resource Studies, 10(2), p.131

http://www.macrothink.org/journal/index.php/ijhrs/article/view/16932/13121 （Accessed 19 February 2021) 